\title{
BMJ Open Efficacy of calcium dobesilate in treating Chinese patients with mild-to-moderate non-proliferative diabetic retinopathy (CALM-DR): protocol for a single-blind, multicentre, 24-armed cluster- randomised, controlled trial
}

Hao Hu, ${ }^{1}$ Jiang Liu, ${ }^{1}$ Duolao Wang (1) , ${ }^{2}$ Shanhu Qiu, ${ }^{1}$ Yang Yuan, ${ }^{1}$ Fenghua Wang, ${ }^{3}$ Liang Wen, ${ }^{4}$ Qi Song, ${ }^{5}$ Zi-lin Sun (iD) ${ }^{1}$

To cite: Hu H, Liu J, Wang D, et al. Efficacy of calcium dobesilate in treating Chinese patients with mild-to-moderate non-proliferative diabetic retinopathy (CALM-DR): protocol for a single-blind, multicentre, 24-armed cluster-randomised, controlled trial. BMJ Open 2021;11:e045256. doi:10.1136/ bmjopen-2020-045256

- Prepublication history for this paper is available online. To view these files, please visit the journal online (http://dx.doi. org/10.1136/bmjopen-2020045256).

$\mathrm{HH}$ and $\mathrm{JL}$ are joint first authors.

Received 25 September 2020 Accepted 07 May 2021

Check for updates

(c) Author(s) (or their employer(s)) 2021. Re-use permitted under CC BY-NC. No commercial re-use. See rights and permissions. Published by BMJ.

For numbered affiliations see end of article.

Correspondence to

Zi-lin Sun;

sunzilin1963@126.com

\section{ABSTRACT}

Introduction Calcium dobesilate $(\mathrm{CaD})$ has been used in the treatment of diabetic retinopathy (DR) due to its potential in protecting against retinal vascular damage. However, there is limited evidence exploring its efficacy in combating DR progression. This study is aimed at evaluating whether $\mathrm{CaD}$ could prevent $\mathrm{DR}$ progression into an advanced stage among Chinese patients with mild-tomoderate non-proliferative DR (NPDR).

Methods and analysis This study is a single-blind, multicentre, cluster-randomised, controlled superiority trial. A total of 1272 patients with mild-to-moderate NPDR will be enrolled and randomly assigned at a 1:1 ratio into the control group (conventional treatment group) and the intervention group (conventional treatment plus $\mathrm{CaD}$ (500 mg three times per day) for 12 months). Patients will be followed at 1, 3, 6 and 12 months after randomisation and receiving treatments, with the severity of DR assessed by the Early Treatment Diabetic Retinopathy Study (ETDRS) scale. The primary endpoint is the progression of $D R$ during follow-up, which is defined as an increase of two or more steps in the ETDRS scale. The secondary endpoints include the concomitant changes in visual acuity, presence, number, location and type of retinal lesions, and retinal blood vessel diameter as well as the arteriovenous ratio at different visits.

Ethics and dissemination Each local ethics committee (first Vote: Ethical Review Committees of Zhongda Hospital of Southeast University (2019ZDSYLL132-P01)) has approved the study. The results will be published in high impact peer-reviewed scientific journals aimed at the general reader.

Trial registration numbers NCT04283162.

\section{INTRODUCTION}

Diabetic retinopathy (DR) is a common microvascular complication associated with diabetes, and encompasses a broad clinical spectrum from the mild non-proliferative DR (NPDR) to a more advanced stage of

\section{Strengths and limitations of this study}

- This will be the first cluster-randomised controlled trial with a large sample size assessing the efficacy of calcium dobesilate (CaD) in treating diabetic retinopathy (DR) among individuals with mild-tomoderate non-proliferative DR.

- This study will collect longitudinal data on patients treated with $\mathrm{CaD}$, and will report on the evolution of several important outcome measures over the first year.

- As with any longitudinal study, there is a risk of loss to follow-up throughout the study period, which may induce bias in the final results.

- A relatively short-term follow-up period of 12 months might also be a limitation for this study.

vision-threatening such as proliferative DR (PDR) and diabetic macular oedema (DME). ${ }^{1}$ The pathogenesis of DR involves a variety of abnormal pathways, which are triggered by a series of factors including chronic hyperglycaemic, free radicals, advanced glycosylation end products, inflammatory cytokines and vascular endothelial growth factors (VEGF). ${ }^{2}$ These may eventually lead to blood-retinal barrier (BRB) breakdown, retinal neovascularisation and retinal neuronal apoptosis, ${ }^{3}$ with BRB injury being considered the hallmark of DR. ${ }^{4}$

There is evidence that $30 \%-50 \%$ of patients with diabetes may develop visionthreatening retinopathy during their lifetime. ${ }^{5}$ Timely intervention, such as intensive diabetes management, adjunctive anti-VEGF therapy, laser photocoagulation and surgical management, can prevent up to $98 \%$ of the cases developing vision loss. ${ }^{1}$ However, tight 
glycaemic control and laser therapy could not reverse the existing ocular damage and may only slow its progression. The safety of ocular surgery and its effectiveness in improving visual acuity have also been questioned. ${ }^{6}$ Moreover, about three-quarters of the world's diabetic patients live in low-income and middle-income countries with severely limited healthcare resources, ${ }^{7}$ which poses a serious challenge to the implementation of anti-VEGF therapy or surgical methods for DR. ${ }^{2}$ Therefore, access to affordable medicines, coupled with early detection of DR, may be a better way to prevent and delay diabetic blindness. ${ }^{1}$

Calcium dobesilate $(\mathrm{CaD})$ is an angioprotective agent that has been proposed as a treatment for DR by protecting against retinal vascular damage. ${ }^{89}$ The earliest clinical study was a 2-year, double-blind, randomised controlled trial (RCT) involving 51 patients and 17 controls. The results showed that $\mathrm{CaD}$ was a potent angioprotector, which can prevent intra and extravascular haemorrhages of retina, reduce the incidence of exudate formation and improve visual acuity. ${ }^{10} \mathrm{~A}$ recent meta-analysis of eight RCTs consisting of 552 patients further demonstrated the angioprotective effects of $\mathrm{CaD}$ on the early stage of DR. ${ }^{11}$ However, other studies have reported that $\mathrm{CaD}$ had no beneficial effect on DR. ${ }^{12-15}$ One of them focused on the effect of $\mathrm{CaD}$ on the development of DME and was followed up for 5 years. This trial concluded that $\mathrm{CaD}$ did not reduce the risk of developing DME. ${ }^{14}$ Therefore, whether $\mathrm{CaD}$ is beneficial to $\mathrm{DR}$ is unclear.

The reason for the discrepancy between clinical trials is unknown. It should be noted that most of these studies generally have small sample size (range from 18 to 194$).{ }^{10121315-21}$ The sample size of such studies is too small to draw any definitive conclusions. Although a most recent study with negative results included a relatively large number of participants, however, the primary endpoint of that study was DME. ${ }^{14}$ As we all know, the first proven effective treatment for DME is macular laser photocoagulation or intravitreal injection of anti-VEGF agent, ${ }^{22}$ rather than $\mathrm{CaD}$. Therefore, inconsistencies in the results of these studies may also be attributable to the inappropriate choice of primary endpoints. A review clearly identified the need for well-designed, robust trials. ${ }^{8}$ We aim to address these problems in the current study.

\section{METHODS AND ANALYSIS}

This study protocol was guided by the Standard Protocol Items: Recommendations for Interventional Trials reporting guidelines. ${ }^{23}$

\section{Trial setting}

The Efficacy of CALcium dobesilate in treating Chinese patients with Mild-to-moderate non-proliferative Diabetic Retinopathy (CALM-DR) is a multicentre randomised controlled study set in 24 tertiary hospitals in China. These centres are located in areas that spans the range of population densities, urbanisation and socioeconomic development status in China. We will recruit patients between June 2021 and December 2021. Follow-up will be completed in December 2022.

\section{Rationale for using CaD}

Despite of the inconsistent results of its efficacy, CaD still remains the only angioprotective agent that reduces the progression of DR. ${ }^{24}$ It was suggested in a review that some double-blind trials relating to permeability variables had better results with $\mathrm{CaD}$ than placebo, in particular a greater reduction in haemorrhage surface area and in fluorescein penetration into the vitreous body. ${ }^{25}$ Others showed an improvement of visual acuity after $\mathrm{CaD}$ treatment. ${ }^{25}$ Another review showed that $\mathrm{CaD}$ had significantly superior effects on the evolution of BRB permeability compared with placebo. ${ }^{24} \mathrm{~A}$ recent meta-analysis indicated that $\mathrm{CaD}$ could effectively treat $\mathrm{DR}$ at the systematic and local ocular levels compared with control. ${ }^{11}$

\section{Safety}

Safety profiles of $\mathrm{CaD}$ have been well established. Side effects are rare and uncommon with therapeutic doses of $\mathrm{CaD}$. Uncommon side effect $(0.1 \%-1 \%)$ is tachycardia. Rare side effects $(0.01 \%-0.1 \%)$ include nausea, diarrhoea, vomiting, rash, fever, chills, arthralgia, and agranulocytosis.

\section{Hypotheses}

In relation to the overall objective, the following are the hypotheses: (1) patients with diabetes who take CaD can reduce the progression of mild-to-moderate DR; (2) this intervention can also improve visual acuity and retinal vascular function of these patients.

\section{Objectives}

The primary objective of this study is to assess the potential therapeutic effect of $\mathrm{CaD}$ on the progression of mildto-moderate DR in adults with diabetes. The secondary objective is to investigate the effect of the intervention on visual acuity and retinal vascular changes in these patients.

\section{Endpoints}

\section{Primary endpoint}

The primary endpoint is the progression of DR during 12 months after randomisation. After 12-month treatment, two digital fundus photographs will be taken for each eye according to Early Treatment Diabetic Retinopathy Study (ETDRS) scale standard of fundus photo description to evaluate the rate of improvement in the progression of patients with mild-to-moderate DR. DR progression is defined as an increase of two or more steps on the ETDRS scale during follow-up. ${ }^{26}$ The progression of mild-tomoderate DR has been widely used in some well-known clinical studies of DR, for example, Diabetes Control and Complications Trial (DCCT) study, Action to Control Cardiovascular Risk in Diabetes (ACCORD) study and 
Wisconsin Epidemiologic Study of Diabetic Retinopathy (WESDR) study. ${ }^{1}$

\section{Secondary endpoints}

1. Progression of DR from baseline to 3, 6 and 12 months.

2. Time from randomisation to the occurrence of progression of DR.

3. ETDRS scale at baseline to 3, 6 and 12 months.

4. Changes in eyesight postintervention from baseline to $1,3,6$ and 12 months/.

5. Changes in the numbers, location and types of the retinal lesions postintervention from baseline to 3,6 and 12 months.

6. Changes in the retinal blood vessel diameter and arteriovenous ratio from baseline to 3,6 and 12 months.

7. Changes in metabolic biomarkers such as HbAlc postintervention from baseline to 3, 6 and 12 months.

Additional repeat testing at 9 months is warranted for patient with type 1 diabetes.

\section{Safety endpoints}

1. Changes in physical examination and vital signs before and after treatment.

2. Changes in laboratory parameters (liver, renal function, blood routine, etc.).

3. Adverse events/serious adverse events and their severities.

\section{Selection of patients}

Inclusion criteria

1. Being diagnosed with mild-to-moderate DR.

2. Being older than 18 years.

3. Being willing to participate in the trial.

\section{Exclusion criteria}

1. Being allergic hypersensitive to experimental drugs or comparator drugs.

2. Having alanine aminotransferase or aspartate aminotransferase $\geq 2$ times higher than the upper limit of normal value, or total bilirubin $\geq 1.5$ times higher than the upper limit of normal value on the exclusion of mild fatty liver disease.

3. Having severe renal insufficiency (defined as an eGFR $\leq 30 \mathrm{~mL} / \mathrm{min} / 1.73 \mathrm{~m}^{2}$ ).

4. Having malignant tumour and some other lifethreatening diseases.

5. Being pregnancy, expecting pregnancy, or breast feeding.

6. Being with unstable conditions, such as: (1) uncontrolled high blood pressure (eg, BP $>180 / 100 \mathrm{~mm}$ $\mathrm{Hg}$ ); (2) glycosylated haemoglobin ( $\mathrm{HbAlc})>8.0 \%$ or uncontrolled high blood glucose or hypoglycaemic; (3) acute cardiovascular events like unstable angina, congestive heart failure, stroke, transient ischaemic attack or myocardial infarction within the previous 3 months; (4) uncontrolled infection and (5) diabetic ketoacidosis or hyperosmolar state in the past 1 month.
7. Being with glaucoma, cataracts or other opacities that may interfere with retinal examination and fundus photography.

8. Receiving drugs such as $\mathrm{CaD}$ or traditional Chinese medicine that may help to improve micro-vascular function in the past 2 weeks.

9. Receiving VEGF therapy in the past 4 months or will be judged to take VEGF therapy because of disease progression.

10. Having attended other clinical trials in the past 1 month, being attending some clinical trials or some other conditions that are unfit for this trial judged by investigators.

Although the recruitment target is mainly middle-aged diabetic patients, the age standard is set at $\geq 18$ years to include young patients with type 1 diabetes, because of the prevalence of DR in type 1 diabetes after an average of 23 years is $77 \%-95 \%$ despite adequate treatment for diabetes. ${ }^{27}$ The majority of exclusion criteria are based on reducing the risk of adverse effects associated with the intervention, as well as the rate of loss of follow-up.

Because some drugs (such as corticosteroids, nonsteroidal anti-inflammatory drugs, antioxidants, inflammatory molecule inhibitors, renin-angiotensin system blockers and fenofibrate) may have an effect on $\mathrm{DR},{ }^{6}$ patients who have used these medicines within 1 month will also be excluded.

\section{Number and source of subjects}

It is expected that approximately 1272 patients will be enrolled into the study from the 24 centres (hospitals) in China.

\section{Screening procedures and prerandomisation investigations}

The scope of our study presents a variety of potential barriers to participant recruitment, for which we will use an assortment of recruitment methods. Our recruitment strategies will rely on community-level advertising, primary health service centre referrals and word-ofmouth recommendations of patients treated in centre in order to inform prospective participants of our study. Given that the focus of this study is on the treatment of $\mathrm{DR}$, it is important to provide potential participants with an assessment of their retinopathy. We also ensure that participation in treatment conditions and data collection are convenient for the participants. A member of each centre research team will be in contact with people interested in participating in the study, screening for eligibility and explaining the main requirements for participation in the study. Written information will be sent to the potential participant who are eligible for participation. Researchers in each hospital will obtain informed consent signed by the participants.

No research-related procedures will be conducted until detailed written information about the study is provided to participants and informed consent is obtained. Those who are still interested in participating in this study after reading the materials will be invited to a health 
examination (visit 0 ): (1) recording of lifestyle characteristics, including smoking and alcohol; (2) recording of medical history, including glucose-lowering medications taken within the last 6 months and other prescription-only medications taking or have taken in the last month; (3) physical examination, including measurement of height, body weight, waist and hip circumferences and vital signs; (4) laboratory measurements of blood specimens and (5) a comprehensive eye examination (see below). Lifestyle education will also be provided.

Recruitment of patients will operate for 12 months in total in each centre.

\section{Randomisation and masking}

Extensive consultation has been conducted among clinical stakeholders in each potential centre to ensure a willingness to be randomised to one of two interventions and agreement to implement the allocated plan on a hospital wide level. Randomisation will be opened to participants and investigators for safety and practical considerations, but researchers related to participant's identification, informed consent acquisition, enrolment and outcome evaluation will be blinded about the treatment allocation. 24 hospitals will be randomised to the intervention and control arms on a 1:1 allocation ratio using a permutedblock randomisation method with block size of four. The allocation sequence will be generated using SAS PROC PLAN independent of the investigators, by the Global Health Trials Unit at the Liverpool School of Tropical Medicine (UK). This is achieved by the study team sharing a list of centres, each of which has a corresponding number. Allocation status will be communicated to the research team coordinator, who will inform respective centres after the investigators obtain the consents of the participants to participate in the trial. To ensure that all baseline measurements of allocated interventions by the investigators are unbiased, the allocation of the intervention groups will be made at the end of the baseline examination (day 6$)$.

\section{Treatment of patients \\ Control group}

Participants allocated to the control group are requested not to alter their original lifestyle habits and treatment plan after randomisation and at the end of the study, with reviews by a study doctor and nurse. Management of blood glucose, blood pressure and lipid profiles is undertaken by the study team during the period of the trial. Any changes to the treatment of these features are made by a doctor who unaware of treatment allocation to minimise the risk of performance bias. During the period of this study, patients are treated as appropriate to maintain the following targets: HbAlc level $<7.5 \%$, blood pressure $<140 / 80 \mathrm{~mm} \mathrm{Hg}$, total cholesterol level $<4.0 \mathrm{mmol} / \mathrm{L}$, triglyceride level $<2.0 \mathrm{mmol} / \mathrm{L}$, high-density lipoprotein level $>1.0 \mathrm{mmol} / \mathrm{L}$ and low-density lipoprotein level $<2.0 \mathrm{mmol} / \mathrm{L}$ (figure 1).

\section{Treatment group}

Patients in the treatment group will receive the same intervention as those in the control group. Additionally, participants will be asked to take a $1500 \mathrm{mg} \mathrm{CaD}$ per day in three divided doses of $500 \mathrm{mg}$ for 1 year. The product will be delivered by the investigator at each centre.

\section{Follow-up of patients}

The duration of follow-up after randomisation is 12 months. Patients will be seen at baseline and subsequently at months 3,6 and 12 by the study team. We have included extra visits at 1 month to the patients' routine schedule of visits. This extra visit is intended to re-enforce adherence to study drugs, to re-enforce their understanding of side effects and the actions that they need to take if these occur, and to examine the participant clinically.

\section{Recording and monitoring of adverse events}

Adverse events will be recorded by the attending physicians and recorded details checked by an independent clinically qualified monitor.

\section{Measures of adherence}

Participants in $\mathrm{CaD}$ treatment will be asked to bring their study medication to visit 2 (month 1 ), visit 3 (month 3), visit 4 (month 6 ) and visit 5 (month 12). The investigators will examine and register the amounts of tablets taken and thereby assess compliance to the drug. A compliance rate $\geq 80 \%$ is considered satisfactory.

\section{Schedule for follow-up/flow chart}

Eligible participants after screening will be included in the study (visit 1-visit 5) (table 1 and figure 1). Study visits are scheduled for the morning, after an overnight fast of $\geq 8$ hour and omission of any morning doses of medications. As mentioned above, during the period of randomised treatments, visits are scheduled to occur at baseline and then after 1, 3, 6 and 12 months ( \pm 7 days). For those who are no longer receiving randomised treatments, they are still scheduled to visit every 6 months, starting from the baseline visit.

At the baseline visit (visit 1), physical examination and blood sampling are repeated, and the following process are completed: (1) first-morning urine sample for measurement of urinary albumin/creatinine ratio; (2) visual acuity; (3) retinal photographs; (4) an examination of concomitant medications and (5) a review of current dietary and physical exercise.

Routine assessments to be performed when patients receive randomised treatment include: (1) complete physical examination; (2) laboratory test of fasting blood samples; (3) urinary albumin/creatinine ratio; (4) visual acuity; (5) retinal photographs; (6) recording of concomitant medications; (7) recording of adverse events and (8) a review of current dietary and physical exercise. 


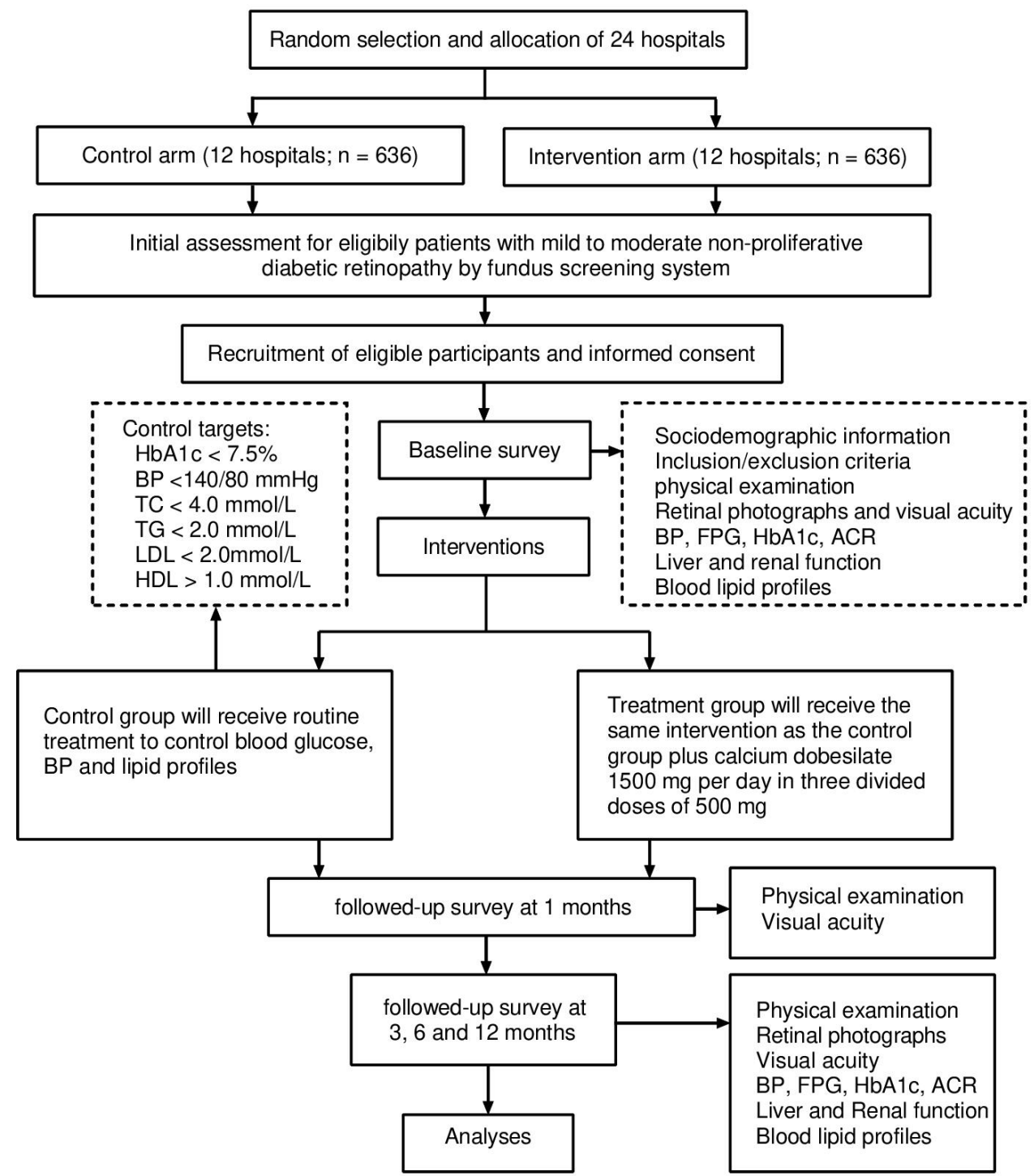

Figure 1 Treatment of patient flow chart. ACR, urinary albumin/creatinine ratio; BP, blood pressure; FPG, fasting plasma glucose; HbA1c, glycosylated haemoglobin; HDL, high-density lipoprotein; LDL, low-density lipoprotein; TC, total cholesterol, $\mathrm{TG}$, triglyceride.

\section{Public and patient involvement}

Patients and/or the public were not involved in the design, or conduct, or reporting, or dissemination plans of this research.

\section{Implementation quality and intervention fidelity}

To ensure both the quality and motivation, a series of dialogue meetings between project partners were held before and after recruitment of hospitals. Meetings between the project and hospital leaderships, and the intervention implementers were held before and after recruitment, and regular meetings are planned throughout the study period. To ensure intervention fidelity, quarterly gatherings will be organised throughout the project period separately for the hospital leaderships and the study group members of each hospital to share experiences and motivate each other.

In each gathering, each hospital is required to develop an execution plan for their specific work the coming quarter. In addition, representatives from project leaderships will phone calls with each team every other week to discuss progress, challenges and solutions.

\section{Assessments}

Anthropometric

Height and body weight are measured in light indoor clothing without shoes. Height and weight are measured to the nearest $0.1 \mathrm{~cm}$ and $0.1 \mathrm{~kg}$, respectively. Waist circumference, the mid-way between lowest rib and iliac crest, hip circumference and the level of the great trochanters, will be measured to the nearest $0.1 \mathrm{~cm}$. Blood pressure measurements are taken after at least $5 \mathrm{~min}$ of rest in duplicate separated by at least $5 \mathrm{~min}$. The mean value of the two measurements will be used in the analysis. Subjects are required to refrain from smoking or ingesting caffeine for 30 min prior to the examination. 


\begin{tabular}{|c|c|c|c|c|c|c|c|}
\hline Visit & Visit 0 & Visit 1 & Visit 2 & Visit 3 & Visit 4 & Visit* & Visit 5 \\
\hline $\begin{array}{l}\text { Time (days from the start of } \\
\text { intervention) }\end{array}$ & -14 & -6 & 30 & 90 & 180 & 270 & 360 \\
\hline Informed consent & $x$ & & & & & & \\
\hline History & $x$ & & & & & & \\
\hline Inclusion/exclusion criteria & $x$ & & & & & & \\
\hline Height & $x$ & $x$ & $x$ & $x$ & $x$ & $x$ & $x$ \\
\hline Body weight & $x$ & $x$ & $x$ & $x$ & $x$ & $x$ & $x$ \\
\hline Waist circumference & $x$ & $x$ & $x$ & $x$ & $x$ & $x$ & $x$ \\
\hline Hip circumference & $x$ & $x$ & $x$ & $x$ & $x$ & $x$ & $x$ \\
\hline Blood pressure & $x$ & $x$ & $x$ & $x$ & $x$ & $x$ & $x$ \\
\hline Glycosylated haemoglobin & $x$ & $x$ & & $x$ & $x$ & $x$ & $x$ \\
\hline Fasting plasma glucose & & $x$ & & & & & $x$ \\
\hline Liver function & $x$ & $x$ & & $x$ & $x$ & $x$ & $x$ \\
\hline Renal function & $x$ & $x$ & & $x$ & $x$ & $x$ & $x$ \\
\hline Blood lipid profiles & & $x$ & & $x$ & $x$ & $x$ & $x$ \\
\hline Urinary albumin/creatinine ratio & & $x$ & & $x$ & $x$ & $x$ & $x$ \\
\hline Fasting blood samples & & $x$ & & & & & $x$ \\
\hline Urine samples & & $x$ & & & & & $x$ \\
\hline Visual acuity & & $x$ & $x$ & $x$ & $x$ & $x$ & $x$ \\
\hline Retinal photographs & $x$ & $x$ & & $x$ & $x$ & $x$ & $x$ \\
\hline Retinal vascular calibre & & $x$ & & $x$ & $x$ & $x$ & $x$ \\
\hline Adverse events & & & $x$ & $x$ & $x$ & $x$ & $x$ \\
\hline Drug accountability & & $x$ & $x$ & $x$ & $x$ & $x$ & $x$ \\
\hline
\end{tabular}

The maximum allowed time interval between screening (visit 0) and baseline examination (visit 1) will be 2 weeks (=14 days). Otherwise, a new screening will be conducted before the participants are included in the study.

${ }^{*}$ Additional visit at 9 months is warranted for patient with type 1 diabetes.

\section{Sociodemographic}

Sociodemographic information, including age, gender, ethnicity, civil status, education, occupation, income, health history, smoking status and alcohol consumption, will be collected at baseline.

\section{Fasting blood and first-morning urine samples}

Fasting blood and first-morning urine samples will be collected and analysed for fasting plasma glucose, HbAlc, liver and renal functions, blood lipid profiles and urinary albumin/creatinine ratio at baseline and follow-up visits (table 1).

\section{Visual acuity}

Visual acuity will be measured in both eyes using ETDRS visual acuity charts at $4 \mathrm{~m}$ by optometrists at baseline and follow-up visits (table 1).

\section{Retinal photographs}

All persons with diabetes attending this study will undergo routine digital retinal photography, which is conducted in a darkened room using a non-mydriatic digital camera capturing optic disc and macular centred images per eye without the use of mydriasis by trained and certified photographers.

According to a grading protocol modified by Airlie House Classification system used in the Multi-Ethnic Study of Atherosclerosis, all retinal images from the patients will be independently reviewed and graded by one of three senior retinal graders who do not know the clinical details. ${ }^{28}$ Levels of DR are classified as no DR (levels 10-13) if no lesions are detected, and any DR (levels 14-80) when at least one microaneurysm and/or a blot haemorrhage are detected. DR is further divided into minimal-moderate NPDR (levels 14-20), mild-moderate NPDR (levels 31-41) and severe NPDR to proliferative retinopathy (levels 51-80). DME is defined as retinal thickening or hard exudates at least one disc diameter to the centre of the macular. Clinically significant macular oedema (CSME), introduced by ETDRS, is defined as DME meeting at least one of three criteria: thickening at or within $500 \mu \mathrm{m}$ of the foveal centre, hard exudates within $500 \mu \mathrm{m}$ of foveal centre with adjacent thickening or at least one disk diameter of thickening with part of it located within one disc diameter of foveal centre. These are confirmed with central macular 
thickness measurements by optical coherence tomography using the macular thickness cube scan protocol. Vision-threatening retinopathy is defined as the presence of severe NPDR, proliferative retinopathy or CSME. The level of retinopathy is graded based on the worse eye. If an eye is ungradable, the score for the other eye will be used to define these outcomes.

Any discrepancies between the two initial graders will be adjudicated by a senior grader using standardised edit rules.

\section{Retinal vascular calibr}

Retinal vascular calibre is measured using a computerbased programme following a previously validated protocol. ${ }^{29}$ Optic disc-centred photographs of the right eye of each participant are measured. Left eye measurements are performed when photographs of the right eye are ungradable. For each photograph, all arterioles and venules coursing through an area 0.5 to 1 -disc diameter from the optic disc margin are measured and summarised as the average central arteriolar and venular equivalents. These equivalents represent the average of projected calibres for the central retinal vessels, and have a high intergrader repeatability with intragrader and intergrader intraclass correlation coefficients ranging from 0.78 to $0.99 .^{30}$

\section{Procedures for assessing safety}

Throughout the course of the study, a steering committee meets to review progress every 6 months, and an independent data safety monitoring board (DSMB) has been established to monitor safety and outcomes. The investigators are responsible for ensuring that all serious adverse events are reported timely to the sponsor, who will then notify the ethics committee of the corresponding centres and the Chinese Medicines Agency according to the current laws and ICH/GCP guidelines. In case of unexpected severe adverse reactions to medication during the study, the trial will be discontinued. In addition, the DSMB may also recommend termination of the study for other serious safety reasons.

Subjects who withdraw from the study for any reason at any time will not be replaced. Subjects who are excluded or who decide to stop participating will be referred to their ophthalmologist for advice on how to manage their DR. At the end of the study, data on withdrawn subjects will be collected and used for efficacy and safety analyses.

\section{Loss to follow-up}

All subjects will be followed up for 12 months duration of the trial. Participants will be sent text message reminders prior to their appointment. If they fail to show, then they will be contacted by phone or by home visit if phoning is not possible to understand the reasons and reschedule another appointment within a week.

\section{Trial closure}

Study follow-up will be for 12 months following randomisation. The trial will be considered closed after the last patient enrolled has completed 12 months of follow-up.

\section{Withdrawal from trial intervention}

Participants can withdraw from the intervention at any time. Participants may be withdrawn from the trial at the discretion of the investigator due to a safety concern or a serious violation of the protocol. Participants will be withdrawn in occurrence of pregnancy or at the intention to become pregnant. Withdrawn participants will be invited for the following assessments every 6 months unless written consent is withdrawn: (1) complete physical examination; (2) fasting blood specimens; (3) visual acuity; and (4) retinal photographs.

\section{Statistical methods}

Sample size

Progression of DR assessed by an increase of two or more steps on the ETDRS severity scale has not previously been used as primary outcome in RCTs of patients with $\mathrm{DR}$ treated with $\mathrm{CaD}$. A prospective observational cohort study showed that with conventional treatment, the proportion of patients with two-step or greater progression of DR at 1 year was $15.2 \%$ (we contacted the study investigators to clarify the unpublished estimate) ${ }^{31}$ It is expected that combined medical therapy of DR in addition to conventional treatment will reduce the development or progression of DR by approximately $50 \%$. ${ }^{1}$ With a two-sided significance level of $5 \%$, a power of $80 \%$ and an intracluster correlation coefficient of $0.01,{ }^{32}$ a clinically meaningful difference in the progression of DR over the 12 -month intervention of $\geq 7.6 \%$ between two groups can be detected with 528 participants in each group. To allow for dropouts as $20 \%$, we plan to include 1272 subjects (636 in each of the two study groups), or 53 in each cluster.

\section{Data management}

Subjects will be identified by study ID. Study data will be collected and managed using the Smart CR secure web-based system ${ }^{33}$ developed by Suzhou MetroHealth Medical Technology, where electronic case report forms will be created. During the intervention phase, data will be entered directly into the Smart CR by study personnel and will be extracted by the investigators or sponsor. The fundus photograph data will be transmitted electronically from the person performing the operation to the same certified photographic grader and will be archived on a secure hard drive with backed up in Southeast University. All blood and urine samples obtained will be stored in a biobank for future use. Samples will be labelled with a unique study identifier.

All collected data will only be used for the purposes of the present study. Participants' information will be confidential and anonymised and will only be treated at a collective level. Trial results will be shared with both the 
scientific community and health professionals, through publications in scientific peer-reviewed journals and presentations at national and international conferences.

\section{Statistical analysis}

All statistical analyses will be performed using SAS V.9.4 and Stata V.14 based on intention-to-treat principle and in accordance with the Consolidated Standards of Reporting Trials (CONSORT) guidelines for reporting cluster randomised trials. Per-protocol analysis will also be performed as supplementary analysis.

A generalised linear mixed-effects model (GLMMIX) will be fitted to analyse the primary endpoint. The model will have a binomial distribution and logit link function and include treatment, time and interaction between treatment and time as fixed factors; baseline ETDRS severity scale as covariate, and cluster and subject as random effects. OR with its $95 \% \mathrm{CI}$ at each time point (3, 6 and 12 months) will be derived, which will also be converted into risk ratio using the mathematical relationship between OR and risk ratio. ${ }^{34}$ A covariate-adjusted analysis of the primary endpoint will be performed by adding prespecified covariates at baseline into GLMMIX. Missing efficacy data will be treated as missing at random and no imputation will be made, because the GLMMIX model is a likelihood-based procedure and handles missing at random as ignorable. ${ }^{35}$ To evaluate the sensitivity of the result of this assumption, the multiple imputation method will be used to impute missing primary endpoint during follow-up. Prespecified subgroup analyses are performed to explore the influence of covariates on primary endpoint.

For secondary binary outcomes, the similar GLMMIX will be used. For secondary continuous outcomes, the change from baseline for each of the outcomes will be modelled using GLMMIX with normal distribution and identity link function and with treatment, time and interaction between treatment and time as fixed factors, baseline measurement as covariate, and cluster and subject as random effects. Mean difference and its $95 \% \mathrm{CI}$ at each time point will be derived. Time-to-event outcome will be analysed using Cox proportional hazards regression model with frailty at the centre (cluster) level and treatment as the study variable and intervention effects will be reported as HR with 95\% CI. Kaplan-Meier plots will also be produced for the two study arms separately and logrank test used to compare two time-to-event curves.

Detailed statistical analyses will be described in the statistical analysis plan, which will be developed and finalised before the database lockup.

\section{ETHICS AND DISSEMINATION}

The study is carried out in accordance with the Helsinki Declaration after approval by each local ethics committee (first Vote: Ethical Review Committees of Zhongda Hospital of Southeast University (2019ZDSYLL132-P01)).
$\mathrm{CaD}$, which has been discovered more than 40 years ago and is registered for the treatment of DR in more than 20 countries remains, to date, is the only angioprotective agent that reduces the progression of this disease. ${ }^{24}$ Although $\mathrm{CaD}$ is effective in animal and/or in vitro models, however, the results of clinical trials are inconsistent. A large, multicentre study is warranted to provide a definitive conclusion. ${ }^{11}$ To the best of our knowledge, this study is the largest multicentre RCT, and is unique in embedding a cluster-randomised trial design within an intervention framework to study the effectiveness of $\mathrm{CaD}$ treatment on the progression of mild-to-moderate NPDR compared with conventional treatment. The efficacy of the intervention on visual acuity, the presence, number, location and type of retinal lesions and retinal blood vessel diameter as well as arteriovenous ratio will also be tested. Our study will give us an opportunity to explore in depth whether $\mathrm{CaD}$ is beneficial for the treatment of mild-to-moderate DR. A limitation of this study is the single-blinded study. Patients' knowing of their treatment groups may have a psychological impact on the trial results. However, the primary endpoint will be assessed by an independent committee and results regarding the primary endpoint will not be biased.

Even in the case of negative results, this trial will produce a large amount of illuminating data. Investigators will be able to closely monitor progression of DR in both arms during the 12-month follow-up period. If the treatment of $\mathrm{CaD}$ is effective, it will provide an additional therapy option for comprehensive management of the diabetic patients with mild-to-moderate DR.

The results of the trial will be analysed, presented and published as soon as possible at high-impact peerreviewed journals and presented at the international scientific meetings and conferences. Manuscripts will be written in accordance with the CONSORT guidelines for reporting cluster randomised trials.

\section{Author affiliations}

${ }^{1}$ Department of Endocrinology, Zhongda Hospital, Institute of Diabetes, School of Medicine, Southeast University, Nanjing, Jiangsu, China

${ }^{2}$ Department of Clinical Sciences, Liverpool School of Tropical Medicine, Liverpool, UK

${ }^{3}$ Beijing Tongren Eye Center, Beijing Tongren Hospital, Capital Medical University and Beijing Ophthalmology and Visual Science Key Lab, Beijing, China

${ }^{4}$ Department of Ophthalmology, Fushun Eye Hospital, Fushun, China

${ }_{5}^{5}$ Jiangsu Wanbang Biochemical Pharmaceutical Group Co., Ltd, Xuzhou, China

Contributors HH and JL drafted the manuscript. HH, JL, DW, SQ, YY, FW, LW, QS and Z-IS participated in the design and preparation of the study. DW provided statistical analysis support. DW, SQ, YY, FW, LW, QS and Z-IS critically revised the manuscript's drafts. All authors approved the final version of the manuscript.

Funding This work is supported by the Diabetes fund project of Chinese Society of Microcirculation.

Disclaimer The funding body provides funds for employment of research assistants, engagement of statistical support and manuscripts processing fees. It is not involved in the design of the study, and collection, analysis and interpretation of data and in writing of the manuscript.

Competing interests None declared. 
Patient and public involvement Patients and/or the public were not involved in the design, or conduct, or reporting or dissemination plans of this research.

Patient consent for publication Not required.

Provenance and peer review Not commissioned; externally peer reviewed.

Open access This is an open access article distributed in accordance with the Creative Commons Attribution Non Commercial (CC BY-NC 4.0) license, which permits others to distribute, remix, adapt, build upon this work non-commercially, and license their derivative works on different terms, provided the original work is properly cited, appropriate credit is given, any changes made indicated, and the use is non-commercial. See: http://creativecommons.org/licenses/by-nc/4.0/.

ORCID iDs

Duolao Wang http://orcid.org/0000-0003-2788-2464

Zi-lin Sun http://orcid.org/0000-0003-1865-1429

\section{REFERENCES}

1 Solomon SD, Chew E, Duh EJ, et al. Diabetic retinopathy: a position statement by the American diabetes association. Diabetes Care 2017;40:412-8.

2 Wong TY, Sun J, Kawasaki R, et al. Guidelines on diabetic eye care: the International Council of ophthalmology recommendations for screening, follow-up, referral, and treatment based on resource settings. Ophthalmology 2018;125:1608-22.

3 Qiu A-W, Bian Z, Mao P-A, et al. II-17A exacerbates diabetic retinopathy by impairing Müller cell function via ACT1 signaling. Exp Mol Med 2016;48:e280.

4 Leal EC, Martins J, Voabil P, et al. Calcium dobesilate inhibits the alterations in tight junction proteins and leukocyte adhesion to retina endothelial cells induced by diabetes. Diabetes 2010;59:2637-45.

5 Stefánsson E, Bek T, Porta M, et al. Screening and prevention of diabetic blindness. Acta Ophthalmol Scand 2000;78:374-85.

6 Semeraro F, Morescalchi F, Cancarini A, et al. Diabetic retinopathy, a vascular and inflammatory disease: therapeutic implications. Diabetes Metab 2019;45:517-27.

7 Song P, Yu J, Chan KY, et al. Prevalence, risk factors and burden of diabetic retinopathy in China: a systematic review and meta-analysis. J Glob Health 2018;8:01083.

8 Liu J, Li S, Sun D. Calcium dobesilate and micro-vascular diseases. Life Sci 2019;221:348-53.

9 Farsa O. Calcium dobesilate in prevention and treatment of diabetic retinopathy. Diabet Retinopathy 2012;2012:356.

10 Salama Benarroch I, Nano H, Pérez H, et al. Assessment of calcium dobesilate in diabetic retinopathy. A double-blind clinical investigation. Ophthalmologica 1977;174:47-51.

11 Zhang X, Liu W, Wu S, et al. Calcium dobesilate for diabetic retinopathy: a systematic review and meta-analysis. Sci China Life Sci 2015;58:101-7.

12 Daubresse JC, Meunier R, Dumont P. A controlled clinical trial of calcium dobesylate in the treatment of diabetic retinopathy. Diabete Metab 1977;3:27-30.

13 Adank C, Koerner F. Calcium dobesilate in diabetic retinopathy. A retrospective controlled study. Ophthalmologica 1985;190:102-11.

14 Haritoglou C, Gerss J, Sauerland C, et al. Effect of calcium dobesilate on occurrence of diabetic macular oedema (CALDIRET study): randomised, double-blind, placebo-controlled, multicentre trial. Lancet 2009;373:1364-71.

15 Larsen HW, Sander E, Hoppe R. The value of calcium dobesilate in the treatment of diabetic retinopathy. A controlled clinical trial. Diabetologia 1977;13:105-9.
16 Benarroch IS, Brodsky M, Rubinstein A, et al. Treatment of blood hyperviscosity with calcium dobesilate in patients with diabetic retinopathy. Ophthalmic Res 1985;17:131-8.

17 Leite EB, Mota MC, de Abreu JR, et al. Effect of calcium dobesilate on the blood-retinal barrier in early diabetic retinopathy. Int Ophthalmol 1990;14:81-8.

18 Vojnikovic B. Hyperviscosity in whole blood, plasma, and aqueous humor decreased by doxium (calcium dobesilate) in diabetics with retinopathy and glaucoma: a double-blind controlled study. Ophthalmic Res 1984;16:150-62.

19 Vojnikovic B, Doxium VB. Doxium (calcium dobesilate) reduces blood hyperviscosity and lowers elevated intraocular pressure in patients with diabetic retinopathy and glaucoma. Ophthalmic Res 1991;23:12-20.

20 Javadzadeh A, Ghorbanihaghjo A, Adl FH, et al. Calcium dobesilate reduces endothelin-1 and high-sensitivity C-reactive protein serum levels in patients with diabetic retinopathy. Mol Vis 2013;19:62-8.

21 Ribeiro ML, Seres Al, Carneiro AM, et al. Effect of calcium dobesilate on progression of early diabetic retinopathy: a randomised double-blind study. Graefes Arch Clin Exp Ophthalmol 2006;244:1591-600.

22 Moisseiev E, Loewenstein A. Diabetic macular edema: emerging strategies and treatment algorithms. Dev Ophthalmol 2017;60:165-74.

23 Chan A-W, Tetzlaff JM, Gøtzsche PC, et al. Spirit 2013 explanation and elaboration: guidance for protocols of clinical trials. BMJ 2013;346:e7586.

24 Garay RP, Hannaert P, Chiavaroli C. Calcium dobesilate in the treatment of diabetic retinopathy. Treat Endocrinol 2005;4:221-32.

25 Berthet P, Farine JC, Barras JP. Calcium dobesilate: pharmacological profile related to its use in diabetic retinopathy. Int $J$ Clin Pract 1999;53:631-6.

26 Bressler SB, Liu D, Glassman AR, et al. Change in diabetic retinopathy through 2 years: secondary analysis of a randomized clinical trial comparing aflibercept, bevacizumab, and ranibizumab. JAMA Ophthalmol 2017;135:558-68.

27 Hautala N, Hannula V, Palosaari T, et al. Prevalence of diabetic retinopathy in young adults with type 1 diabetes since childhood: the Oulu cohort study of diabetic retinopathy. Acta Ophthalmol 2014;92:749-52.

28 Wong TY, Klein R, Islam FMA, et al. Diabetic retinopathy in a multi-ethnic cohort in the United States. Am J Ophthalmol 2006;141:446-55.

29 Wong TY, Knudtson MD, Klein R, et al. Computer-assisted measurement of retinal vessel diameters in the Beaver dam eye study: methodology, correlation between eyes, and effect of refractive errors. Ophthalmology 2004;111:1183-90.

30 Cheung CY-L, Hsu W, Lee ML, et al. A new method to measure peripheral retinal vascular caliber over an extended area. Microcirculation 2010;17:495-503.

31 Lim LS, Chee ML, Cheung CY, et al. Retinal vessel geometry and the incidence and progression of diabetic retinopathy. Invest Ophthalmol Vis Sci 2017;58:BIO200.

32 Gao F, Earnest A, Matchar DB, et al. Sample size calculations for the design of cluster randomized trials: a summary of methodology. Contemp Clin Trials 2015;42:41-50.

33 Smart CR - an online web-based clinical trial management system, 2019. Available: http://yhx.smart-cr.com

34 Zhang J, Yu KF. What's the relative risk? A method of correcting the odds ratio in cohort studies of common outcomes. JAMA 1998;280:1690-1.

35 Schafer JL. Analysis of incomplete multivariate data. London, United Kingdom: Chapman \& Hall. Press, 1997. 